\title{
Proteomic characterization of the cellular response to chemopreventive triterpenoid astragaloside IV in human hepatocellular carcinoma cell line HepG2
}

\author{
HONGYI QI ${ }^{1,2}$, LAI WEI ${ }^{1,2}$, YIFAN HAN ${ }^{3}$, QINGLIN ZHANG ${ }^{4}$, ALLAN SIK-YIN LAU ${ }^{1}$ and JIANHUI RONG ${ }^{1,2}$ \\ ${ }^{1}$ Molecular Chinese Medicine Laboratory, ${ }^{2}$ School of Chinese Medicine, Li Ka Shing Faculty of Medicine, \\ University of Hong Kong, 10 Sassoon Road, Pokfulam, Hong Kong; ${ }^{3}$ Department of Applied Biology \\ and Chemical Technology, Hong Kong Polytechnic University, Hung Hom, Kowloon, Hong Kong; \\ ${ }^{4}$ Department of Experimental Hematology, Beijing Institute of Radiation Medicine, Beijing, P.R. China
}

Received June 19, 2009; Accepted September 14, 2009

DOI: 10.3892/ijo_00000548

\begin{abstract}
Triterpenoids are implicated in the chemoprevention of various cancers. Current challenge is to define the molecular mechanism underlying the chemopreventive activity of triterpenoids. This study was designed to characterize the intracellular proteins regulated by astragaloside IV, the major active triterpenoid in Radix Astragali. Upon the treatment with astragaloside IV, human hepatocellular carcinoma HepG2 cells were evaluated for the colonogenic survival and anchorage-independent growth. The cellular proteins of treated and untreated cells were resolved by 2-D polyacrylamide gel electrophoresis. The protein spots mostly altered by drug treatment were identified by mass spectrometry and subsequently verified by Western blotting using specific antibodies and RT-PCR technique using specific DNA primers. We found that astragaloside IV attenuated the colonogenic survival and anchorage-independent growth of cancer cells. Based on the proteomic profiles, top 14 upregulated and 13 down-regulated protein spots were subjected to mass spectrometric analysis. As an example, Vav3.1 belongs to the oncogene Vav family, which is implicated in tumorigenesis. Vav3.1 expression was down-regulated by astragaloside IV in a dose- and time-dependent manner. Down-regulation of Vav3.1 was highly correlated with the suppression of cell malignant transform. Thus, astragaloside IV may elicit anticancer activity via down-regulating the expression of oncogenes such as Vav3.1.
\end{abstract}

Correspondence to: Dr Jianhui Rong, School of Chinese Medicine, Li Ka Shing Faculty of Medicine, University of Hong Kong, 10 Sassoon Road, Pokfulam, Hong Kong, P.R. China

E-mail: jrong@hkucc.hku.hk

Key words: chemoprevention, tumorigenesis, gene regulation, oncogene Vav3, astragaloside IV

\section{Introduction}

Tumorigenesis is a complex pathological process involving three distinct stages of initiation, promotion and progression (1). Although several etiologies exist, aberrant activation of various oncogenes is a key intracellular event in the process of tumorigenesis $(2,3)$. Activated oncogenes disrupt the cellular signals that govern the cell proliferation, differentiation and apoptosis $(4,5)$. Some oncogenes may intervene with the intercellular signal transduction normally mediated by growth factors, neurotrophic factors and cytokines (6-8). For example, a family of three 21-kDa Ras proteins, namely, H-Ras, N-Ras and K-Ras, are often activated in the process of cancer development (7,9-11). Notably, the activity of Ras proteins is highly dependent on the replacement of bound guanosine diphosphate (GDP) with guanosine triphosphate (GTP), an enzymatic reaction catalyzed by a large group of guanine nucleotide exchange factors (GEFs) including SOS-1/2, $\mathrm{CDC}_{25^{\mathrm{Mm}}}$ and Vav proteins (7,10-13). There is compelling evidence supporting that GEFs such as Vav proteins (Vav1, Vav2 and Vav3) are hyperactive in various cancers (14-17). Equally importantly, RNAi-mediated knock-down of Vav3 expression resulted in significant regression of tumor growth $(15,17)$. Recent mechanistic studies demonstrated that Vav proteins activate a panel of small GTPases such as Rho, Rac and Cdc42 (18-21). Active Rho, Rac and Cdc42 subsequently stimulate the formation of focal adhesion complexes involving integrins, paxillin, vincullin and focal adhesion kinase (FAK), and induce membrane ruffling and filopodia formation $(14,20,22)$. Rearrangement of cytoskeleton structure is a key event in cell malignant transformation. In addition, Vav proteins directly interact with Grb2, Shc and the p85 subunit of PI3K and subsequently modulate receptor tyrosine kinase signaling pathways, leading to the activation of Akt, MAPK and Stat3 (23-27). These results highlight the possibility that GEFs including Vav proteins may be therapeutic targets in inhibiting cell oncogenic transformation (23,28-30).

Radix Astragali is a dietary supplement widely used in traditional Chinese medicine to prevent and treat various 
cancers (31). The molecular basis of the anticancer activity of Radix Astragali extract has not been fully explored. Triterpenoid astragalosides and polysaccharides were suggested to be active against cancers $(32,33)$. Recent studies showed that herbal polysaccharides modulate human immune system in a non-specific manner $(34,35)$. The total astragaloside extract of Radix Astragali inhibited the growth of human colon cancer cells in cell culture system and in a xenograft cancer model (33). Astragaloside IV represents a group of cycloartane-type triterpenoid glycosides and is used as a marker compound in Radix Astragali (Fig. 1). Several cycloartane-type triterpenoid glycosides are found to be chemopreventive and structurally related ginsenoside $\operatorname{Rg} 3$ is clinically applied to treat cancers (36). These results motivated us to investigate the anticancer activity of astragalosides with the hope of developing a new cancer therapy.

In the present study, we investigated the effects of astragaloside IV on the colony formation of tumor cells. To characterize the cellular targets for astragaloside IV, we compared the proteomic profiles of human liver cancer cells treated with astragaloside IV or vehicle, respectively. Owing to its potential in tumorigenesis, the regulation of oncogene Vav3.1 by astragaloside IV was further investigated by RT-PCR technique and Western blotting.

\section{Materials and methods}

Cell culture and reagents. Human hepatocellular carcinoma cell line HepG2 was obtained from American Type Culture Collection (Rockville, MD, USA) and cultured in Eagle's minimum essential medium (MEM) containing $10 \%$ fetal bovine serum and supplemented with $100 \mathrm{U} / \mathrm{ml}$ penicillin and $100 \mu \mathrm{g} / \mathrm{ml}$ streptomycin. The cells were maintained in a humidified incubator under $5 \% \mathrm{CO}_{2}$ at $37^{\circ} \mathrm{C}$. Astragaloside IV was obtained from the National Institute for the Control of Pharmaceutical and Biological Products (Beijing, China). For cell culture, a stock astragaloside IV solution $(200 \mathrm{mg} / \mathrm{ml})$ in dimethyl sulfoxide (DMSO) was sterilized by passing through a Costa Spin-X nylon filter (Corning, USA). In order to generate a pharmacologically altered cell type, HepG2 cells were treated with astragaloside IV at the concentration of $150 \mu \mathrm{g} / \mathrm{ml}$ in the complete growth medium MEM for 3 days, whereas an equal volume of DMSO was added to control cultures.

Cell proliferation assay. Following the 3-day treatment with astragaloside IV $(150 \mu \mathrm{g} / \mathrm{ml})$ or vehicle alone, the cells $\left(1 \times 10^{5}\right.$ cells/well) were seeded into three 6 -well plates in the complete growth medium supplemented with astragaloside IV $(150 \mu \mathrm{g} / \mathrm{ml})$ or vehicle alone. Each experiment was performed with three biological replicates. The cells were harvested and counted after incubation for 24,48 and $72 \mathrm{~h}$, respectively.

Clonogenic survival assay. Following the treatment with astragaloside IV $(150 \mu \mathrm{g} / \mathrm{ml})$ or vehicle alone consecutively for 3 days, ASIV-treated and control HepG2 cells were examined for focus formation. The cells (400 cells/well) were seeded into a 6-well plate in MEM supplemented with $5 \%$ fetal bovine serum in the presence of astragaloside IV $(150 \mu \mathrm{g} / \mathrm{ml})$ or vehicle alone. Fourteen days later, the cells were fixed in $70 \%$ ethanol at room temperature for $20 \mathrm{~min}$, air dried for $30 \mathrm{~min}$, and then stained with crystal violet (SigmaAldrich) at $37^{\circ} \mathrm{C}$ for $60 \mathrm{~min}$. After washing two times with $1 \mathrm{X}$ phosphate-buffered saline, cells were air dried and foci were enumerated. The clonogenic survival was calculated by the following equation: clonogenic survival $(\%)=($ clone number/cells seeded) x 100\%. This experiment was performed with three biological replicates.

Anchorage-independent growth in soft agar. Following the treatment with astragaloside IV $(150 \mu \mathrm{g} / \mathrm{ml})$ or vehicle alone consecutively for 3 days, ASIV-treated and control HepG2 cells were examined for the anchorage-independent growth in agarose. The cells were trypsinized and washed twice with serum-free medium, and centrifuged for $5 \mathrm{~min}$ at $1200 \mathrm{rpm}$, and re-suspended at the density of 400 cells $/ \mathrm{ml}$ in the complete growth medium supplemented with $150 \mu \mathrm{g} / \mathrm{ml}$ of astragaloside IV or vehicle alone. The bottom agarose was prepared by mixing equal volume of sterilized $1.2 \%$ agar with $2 \mathrm{X}$ MEM supplemented with $20 \%$ fetal bovine serum at $42^{\circ} \mathrm{C}$, applied into a 6-well cell culture plate by $3 \mathrm{ml}$ per well and cooled down to solid. The remaining agarose was kept at $42^{\circ} \mathrm{C}$ in water, and mixed equivalently with the cell suspension containing $150 \mu \mathrm{g} / \mathrm{ml}$ of astragaloside IV or vehicle alone. The mixture was immediately poured into the cell culture plate at $2 \mathrm{ml}$ per well, 400 cells per well. The cells were cultured at $37^{\circ} \mathrm{C}$ in the incubator equilibrated with $5 \% \mathrm{CO}_{2}$ for 14 days. The clones with a diameter $>50 \mu \mathrm{m}$ were observed and counted under an inverted image difference microscope. The clonogenic fraction was calculated by the following equation: colony formation rate $(\%)=$ (clone number/cells seeded) $\mathrm{x} 100 \%$. This experiment was performed with three biological replicates.

Two-dimensional (2-D) gel electrophoresis. HepG2 cells were treated with astragaloside IV $(200 \mu \mathrm{g} / \mathrm{ml})$ or vehicle alone at $37^{\circ} \mathrm{C}$ for $24 \mathrm{~h}$. At the end of the treatment, the cells were harvested and washed twice with ice-cold PBS. The cells were pelleted and lysed with a $40 \mathrm{mM}$ Tris buffer consisting of $8 \mathrm{M}$ urea, $4 \%$ CHAPS, 2\% ampholine, and $65 \mathrm{mM}$ dithiothreitol (DTT). The cellular proteins were recovered following centrifugation at 20,000 $\mathrm{xg}$ for $60 \mathrm{~min}$ at $4^{\circ} \mathrm{C}$, and protein concentration was measured using the Bradford assay (37). Proteins were separated by 2 -D electrophoresis essentially according to the manufacturer's instructions (Amersham Biosciences, Uppsala, Sweden). For isoelectric focusing (IEF), $300 \mu \mathrm{g}$ of cellular proteins were mixed with a rehydration solution containing $2 \mathrm{M}$ thiourea, $7 \mathrm{M}$ urea, 2\% CHAPS, $0.5 \%$ immobilized-pH-gradient (IPG) buffer ( $\mathrm{pH} 4.0$ 7.0) and $10 \mathrm{mM}$ DTT. The IEF was subsequently carried out in $24 \mathrm{~cm}$ Immobiline Drystrips ( $\mathrm{pH} \mathrm{4.0-7.0)} \mathrm{using} \mathrm{an}$ ETTAN IPGphor 3 apparatus (GE Healthcare). The focusing was achieved sequentially at $50 \mathrm{~V}$ for $24 \mathrm{~h}, 500 \mathrm{~V}$ for $1 \mathrm{~h}$, $1,000 \mathrm{~V}$ for $1 \mathrm{~h}$, and then held at $8,000 \mathrm{~V}$ until current droped $<25 \mu \mathrm{Am}$ and for a total of $>100,000 \mathrm{~V} / \mathrm{h}$. When the IEF was completed, the individual strips were equilibrated in $50 \mathrm{mM}$ Tris-HCl buffer ( $\mathrm{pH} 8.8$ ) containing $6 \mathrm{M}$ urea, 30\% glycerol, $2 \%$ SDS, $1 \%$ DTT and a trace of bromophenol blue. The free thiol groups were inactivated with $2.5 \%$ iodoacetamide in the same buffer for another $20 \mathrm{~min}$. After the IPG strips were 
mounted on the top of the gels, proteins were resolved in $12.5 \%$ SDS gels using the Ettan Dalt six electrophoresis unit (GE Healthcare). SDS-PAGE was run at $20 \mathrm{~W} /$ gel for $4 \mathrm{~h}$ until the bromophenol blue front reached the bottom of the gel.

Protein imaging and analysis. The proteins resolved in the gel were visualized by a standard silver staining procedure described in the manufacturer's instructions (Amersham Biosciences). The stained gels were scanned using ImagerScanner with LabScan software version 5.0 (GE Healthcare) and analyzed using ImageMaster Platinum software version 5.0 (GE Healthcare). Image analysis included spot detection, spot editing, background subtraction, and spot matching. The resulting data were exported to Microsoft Excel for comparison.

In-gel proteolytic digestion. An automated spot-picker was used to recover the spots of interest from the $2 \mathrm{D}$ gels to corresponding siliconized Eppendorf tubes. The silver stain was removed by a freshly prepared destaining solution $[30 \mathrm{mM}$ potassium hexacyanoferrate: $100 \mathrm{mM}$ sodium thiosulfate, 1:1 $(\mathrm{v} / \mathrm{v})]$. After a couple of rinses with water, the gel pieces were incubated in $100 \mathrm{mM}$ ammonium bicarbonate for $5 \mathrm{~min}$, and then dehydrated with $100 \%$ acetonitrile. The residual solvents were removed in a vacuum centrifuge. Proteins were digested by $20 \mu \mathrm{g} / \mathrm{ml}$ proteomics sequencing grade trypsin (Promega) in the ice-cold buffer containing $40 \mathrm{mM}$ ammonium bicarbonate, $9 \%$ acetonitrile. The peptides were extracted twice by adding 5\% formic acid 50\% acetonitrile for $15 \mathrm{~min}$ at room temperature. The resulting solutions were dried in a vacuum centrifuge.

Peptide identification by MALDI-TOF mass spectrometry. The peptide residues were dissolved in $10 \mu \mathrm{l}$ of $0.1 \%$ formic acid. Peptide mixtures $(1 \mu \mathrm{l})$ were then mixed with an equal volume of $10 \mathrm{mg} / \mathrm{ml} \alpha$-cyano-4-hydroxycinnamic acid (Sigma) saturated with $50 \%$ acetonitrile $/ 0.1 \%$ formic acid and spotted onto a MALDI target plate. The peptide spots were analyzed with an ABI 4800 MALDI-TOF/TOF mass spectrometer (Applied Biosystems, Foster City, CA). The MS setting was positive reflector mode. Laser shots of 200 per spectrum were used to acquire spectra with a mass range of 900-4,000 Da with a focus mass of 2,000 Da. The MS/MS setting was $2 \mathrm{kV}$ positive/CID on and 5 monoisotopic precursors selected ( $\mathrm{S} / \mathrm{N}>200)$. The calibration was performed using the Calibration mixture 1 of 4,700 Proteomics Analyzer calibration mixture (Applied Biosystems). The spectra were calibrated externally using P14R and insulin chain B oxidized from bovine pancreas (Sigma). Autolytic peaks of trypsin served as internal standards for mass calibration. A combined database search of PMF and MS/MS data was performed using GPS Explorer ${ }^{\mathrm{TM}}$ Workstation (Applied Biosystems), and the NCBInr database was selected in the MASCOT search engine (www.matrix-science.com). Search parameters included Homo sapiens, trypsin digest (allowed up to 1 missed cleavage), cysteines modified by carbamidomethylation, methionine modified by oxidation, and the mass tolerance was set at $75 \mathrm{ppm}$. Probability scores greater than 63 were defined as significant $(\mathrm{p}<0.05)$. In cases of low score matching, duplicate runs were made to ensure the accuracy of analysis. The protein spots were annotated by searching Gene Ontology (http://www.geneontology.org/).

Western blot analysis. Total cell lysates containing $30 \mu \mathrm{g}$ of proteins were resolved by $12 \%$ SDS-PAGE, and were subsequently transferred onto PVDF transfer membrane (GE Healthcare Life Sciences). Membranes were blocked with $5 \%$ non-fat milk in TBST (50 mM Tris-Cl, pH 7.6, $150 \mathrm{mM} \mathrm{NaCl}$, and $0.1 \%$ Tween-20) at $4^{\circ} \mathrm{C}$ overnight. The membranes were then probed with the antibodies specific for the indicated proteins (1:1,000 dilution) at room temperature for $1 \mathrm{~h}$. The bound antibodies were detected by horseradish peroxidase (HRP)-conjugated secondary antibody $(1: 1,500$ dilution) at room temperature for $1 \mathrm{~h}$ and then visualized by enhanced chemiluminescence (ECL) reaction reagents (GE Healthcare Life Sciences). Protein loading was normalized by re-probing the same membranes with $\beta$-actin specific antibodies.

$R T$-PCR detection. Following the treatment with astragaloside IV or vehicle alone, total RNA was isolated using RNeasy kit (Qiagen, USA) and subsequently converted into cDNA templates by reverse transcription using SuperScript II RT kit and random hexamer primers (Invitrogen, USA). The intracellular mRNAs encoding VAV3.1 (NM_001079874) and B-actin (NM_001101) were determined by PCR technique with AccuPrime ${ }^{\text {TM }}$ Taq DNA polymerase (Invitrogen) and specific primers: VAV3.1 sense: 5'-CCAATTTTTACATTT CTTTCAGA-3', VAV3.1 antisense: 5'-TTATTCATCCTCT TCCACATATG-3'; ß-actin sense: 5'-GGCACCACACCTTC TACAATGA-3', ß-actin antisense: 5'-GGAGTTGAAGGTA GTTTCGTGGA-3'. The resultant PCR products were analyzed by $1.2 \%$ agarose gel electrophoresis.

Statistical analysis. Statistical analysis was performed by Microsoft Excel 2003. All means were calculated from three experiment replicates, and the error bars represent standard error of mean (SEM). All data are expressed as means \pm SEM. Statistical significance was determined by paired and two-tailed Student's t-test. A $p<0.05$ was considered statistically significant.

\section{Results}

Inhibition of tumorigenesis by astragaloside IV. To achieve a pharmacologically altered cell phenotype, HepG2 cells were treated with astragaloside IV for three consecutive days. Astragaloside IV-treated and untreated HepG2 cells were examined for the proliferation, focus formation and anchorageindependent growth in soft agar. Under conventional cell culture conditions, astragaloside IV did not show any alteration on the proliferation of HepG2 cells (data not shown). This result supports the clinical use of astragaloside IV-containing Radix Astragali in a large quantity without causing evident cytotoxicity (38). When the cells were cultured in the growth medium supplemented with $5 \%$ fetal bovine serum, however, astragaloside IV-treated cells showed a dramatic decrease in the colonogenic survival in the culture dishes (Fig. 2A). Astragaloside IV also exhibited an inhibitory effect on the anchorage-independent growth of HepG2 cells in the soft 

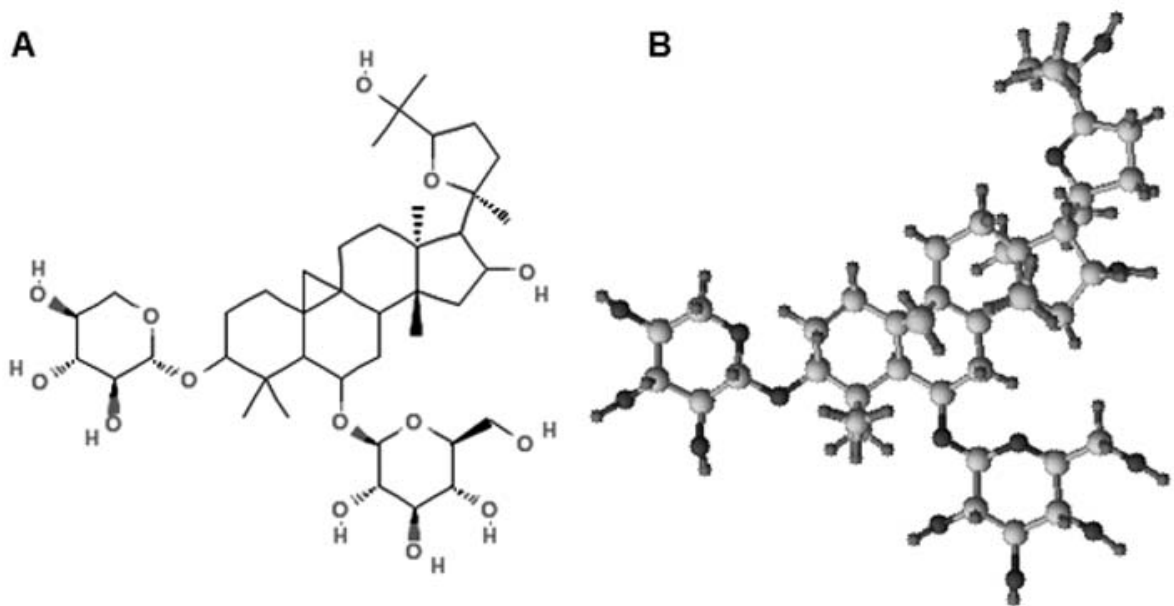

Figure 1. Structure of astragaloside IV. Chemical structure (A) and 3D structure (B) of astragaloside IV were generated by ChemSketch Version 11.01 (http://www.acdlabs.com).

A
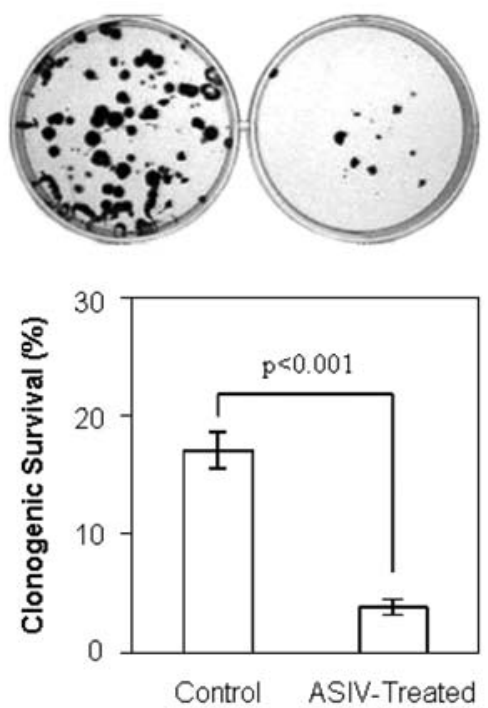
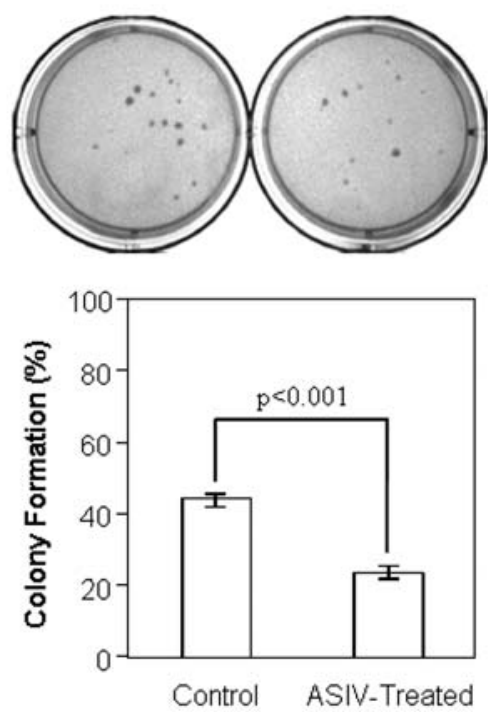

Figure 2. In vitro inhibition of tumorigenesis by astragaloside IV. (A) Clonogenic survival. Following continuous exposure to astragaloside IV or vehicle alone for 3 days, HepG2 cells (400 cells/well) were seeded in 6-well plate in triplicates. The cells were cultured in the presence of 5\% fetal bovine serum for 12 days. The cell foci were stained with $0.5 \%$ crystal violet in $20 \%$ ice-cold methanol as described in Materials and methods. The stained cell foci were then counted under light microscopy. A p $<0.05$ was considered as a significant difference between the two groups. (B) Growth in soft agar. Following continuous exposure to astragaloside IV or vehicle alone for 3 days, HepG2 cells (400 cells/well) were seeded in 6-well plate in triplicates. The cells were cultured in agarose plates supplemented with complete growth medium for 14 days. The cell colonies were counted under inverted phase microscope. A p $<0.05$ was considered as a significant difference between the two groups. 1

agar (Fig. 1B). Importantly, transformed cells are able to survive and grow in an anchorage-independent manner (39). Thus, our results suggest for the first time that astragaloside IV is active in inhibiting the colony formation of HepG2 cells as a surrogate of liver cancer.

Identification of the proteins mostly affected by astragaloside $I V$. To elucidate the molecular mechanisms underlying the inhibition of astragaloside IV on tumorigenesis, we examined the effect of astragaloside IV on the protein expression in human cancer cells. The cellular proteins obtained from astragaloside IV-treated or untreated HepG2 cells were resolved by 2D gel electrophoresis (Fig. 3). After spot detection, spot editing and spot matching, the protein map of astragaloside IV-treated cells was quantitatively compared with that of untreated cells using ImageMaster Platinum software version 5.0 (GE Healthcare). The protein spots were classified into two different groups: up-regulated and downregulated, respectively (Table I). According to the rank of the fold change, 14 upregulated protein spots were picked up from the astragaloside IV-treated samples, whereas 13 downregulated protein spots were obtained from the untreated samples. The selected protein spots were subjected to in-gel digestion and subsequent MALDI-TOF mass spectrometry 


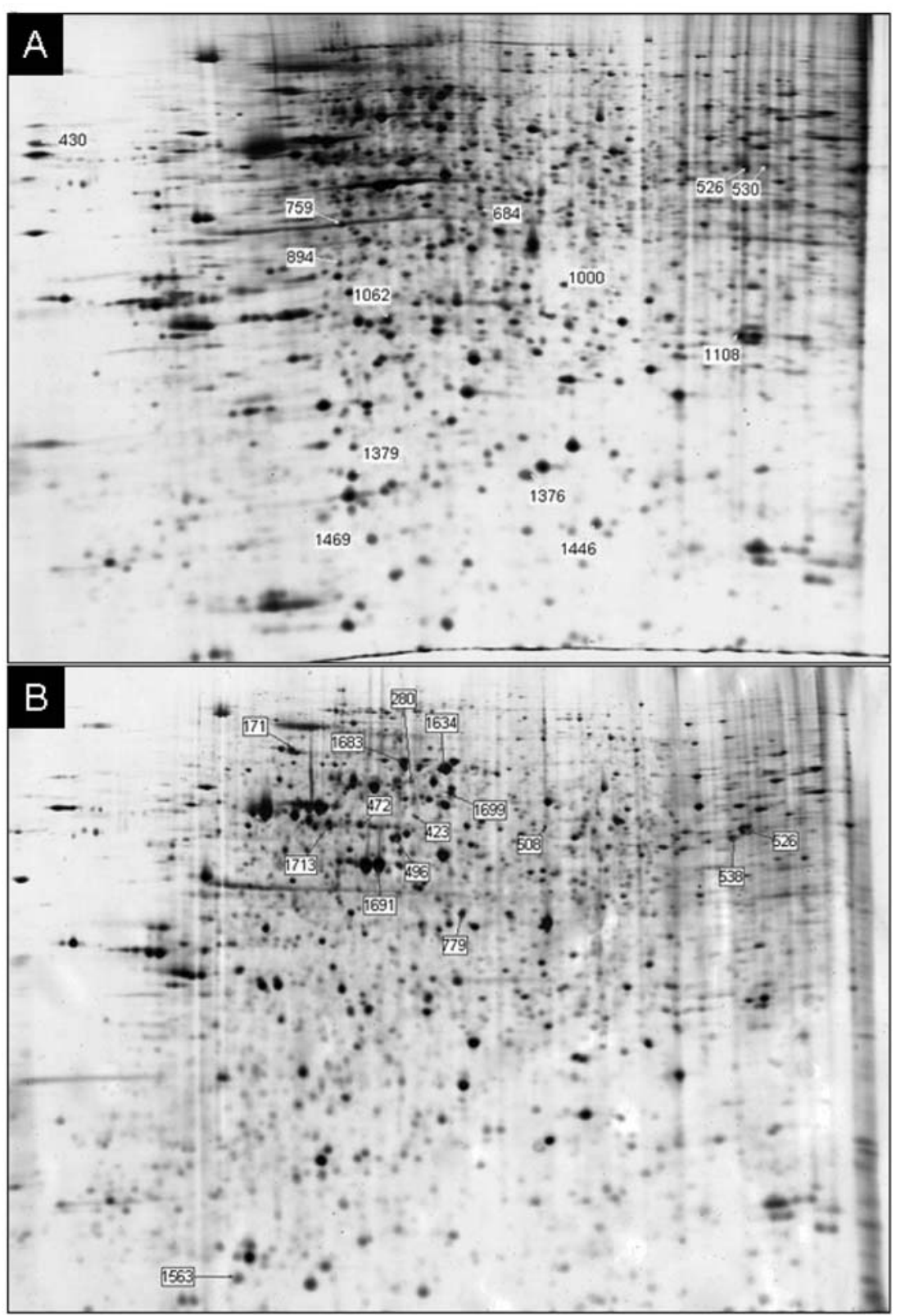

Figure 3. Representative proteome profiles of HepG2 cells with or without exposure to astragaloside IV. The gels are orientated according to the pH gradients on the horizontal axes from $3 \mathrm{pH}$ units (left) to $10 \mathrm{pH}$ units (right) and the approximate apparent molecular mass on the vertical axes ranging from $10 \mathrm{kDa}$ (bottom) to $200 \mathrm{kDa}$ (top). Image A represents the proteome profile of HepG2 cells treated with astragaloside IV whereas image B represents the proteome profile of HepG2 cells treated with vehicle only. The most altered protein spots are highlighted by arrows and the index number of individual spots in the reference gel is indicated in the rectangles. The up-regulated and down-regulated protein spots were excised from astragaloside IV and vehicle-treated samples, respectively.

analysis. The protein identities corresponding to individual spots were annotated using Mascot software (http://www. matrixscience.com) against public protein database (NCBInr).

As shown in Table I, the cellular responses to astragaloside IV treatment can be classified into three functional categories: up-regulated group, up/down-regulated group and down-regulated group, respectively. Firstly, astragaloside IV appeared to enhance the cellular response to stress stimuli. Several stress responsive proteins such as glucose-regulated protein BiP/GRP78 (NP _005338), heat shock $70 \mathrm{kDa}$ protein 2 (NP_068814), HSPA1A protein (NP_005336) and HSPA8 protein (NP_006588) and chaperon containing TCP1, subunit $8(\theta)$ (NP_006576), belong to the up-regulated group. It is interesting that several astragaloside IV-affected proteins are involved in protein synthesis, especially the initiation stage.
Eukaryotic translation initiation factor 3S2b (NP_003748) and eukaryotic translation initiation factor 4A1 (NP_001407) are also upregulated. Moreover, proliferation associated $2 \mathrm{G} 4$ protein (AAH32111) is an RNA-binding protein that is involved in the regulation of cell growth (40). Our results also showed that the expression of proliferation-associated proteins was enhanced by astragaloside IV. Secondly, the proteins involved in cytoskeleton organization and protein degradation were either positively or negatively regulated by astragaloside IV. For example, structural proteins including desmin (NP_001918), keratin 5 (NP_000415), tubulin $\alpha$-1B (P68363), ß-actin (NP_001092) and cytokeratin (NP_002264) were upregulated up to 5.8 -fold, whereas a few others such as keratin 86 (NP_002275), plectin (NP_000436), cytokeratin type II (NP_004684) and outer dense fiber of sperm tails 2-like 
Table I. MALDI/MS identification of the protein spots with largest expression changes in response to astragaloside IV.

\begin{tabular}{|c|c|c|c|c|c|c|c|c|}
\hline \multirow[b]{2}{*}{$\begin{array}{c}\text { Spot } \\
\text { no. }\end{array}$} & \multirow[b]{2}{*}{$\begin{array}{c}\text { Fold } \\
\text { change }\end{array}$} & \multirow[b]{2}{*}{ MW (Da) } & \multirow[b]{2}{*}{$\mathrm{pI}$} & \multicolumn{5}{|c|}{ Mascot search results } \\
\hline & & & & Protein description & Accession no. & $\begin{array}{l}\text { Peptide } \\
\text { matches }\end{array}$ & $\begin{array}{c}\text { Coverage } \\
(\%)\end{array}$ & $\begin{array}{c}\text { Protein } \\
\text { scores }\end{array}$ \\
\hline 171 & 8.8 & 72402.5 & 5.07 & Glucose-regulated protein BiP/GRP78 & NP_005338 & 13 & 24.5 & 202 \\
\hline 508 & 5.8 & 53544.2 & 5.21 & Desmin & NP_001918 & 15 & 23.9 & 61 \\
\hline 1713 & 4.7 & 54682.3 & 5.32 & Proteasome 26S ATPase subunit 3 & NP_002795 & 16 & 26.2 & 156 \\
\hline 779 & 4.0 & 36877.8 & 5.38 & Eukaryotic translation initiation factor $3 \mathrm{~S} 2 \mathrm{~b}$ & NP_003748 & 11 & 34.2 & 85 \\
\hline 280 & 3.3 & 62568.1 & 7.59 & Keratin 5 & NP_000415 & 16 & 23.7 & 62 \\
\hline 423 & 2.9 & 50803.9 & 4.94 & Tubulin $\alpha-1 \mathrm{~B}$ & P68363 & 5 & 12.2 & 83 \\
\hline 538 & 2.6 & 41996.4 & 7.14 & Proliferation associated $2 \mathrm{G} 4$ protein & AAH32111 & 6 & 27.3 & 74 \\
\hline 1691 & 2.5 & 40536.2 & 5.55 & $\beta$-actin & NP_001092 & 16 & 49.7 & 406 \\
\hline 1683 & 2.4 & 70263.0 & 5.56 & Heat shock $70 \mathrm{kDa}$ protein 2 & NP_068814 & 12 & 22.5 & 212 \\
\hline 496 & 2.3 & 46352.6 & 5.32 & Eukaryotic translation initiation factor $4 \mathrm{~A} 1$ & NP_001407 & 13 & 26.6 & 202 \\
\hline 1634 & 2.3 & 70294.1 & 5.48 & HSPA1A protein & NP_005336 & 19 & 53.0 & 514 \\
\hline 472 & 2.1 & 64804.2 & 5.36 & HSPA8 protein & NP_006588 & 16 & 25.7 & 234 \\
\hline 1699 & 2.1 & 60152.7 & 5.42 & Chaperon containing TCP1, subunit $8(\theta)$ & NP_006576 & 13 & 23.5 & 141 \\
\hline 526 & 2.1 & 59779.1 & 7.60 & Cytokeratin & NP_002264 & 13 & 21.6 & 62 \\
\hline 1379 & -1.7 & 32604.2 & 9.01 & VAV-3 protein $B$ isoform & NP_001073343 & 5 & 15.1 & 58 \\
\hline 1469 & -1.8 & 10638.6 & 9.15 & Stomatin (EPB72)-like 2 & NP_038470 & 7 & 20.5 & 57 \\
\hline 1108 & -2.3 & 25133.2 & 6.00 & Peroxiredoxin 6 & NP_004896 & 8 & 37.1 & 100 \\
\hline 1330 & -2.3 & 55119.9 & 5.56 & Keratin 86 & NP_002275 & 11 & 20.2 & 48 \\
\hline 684 & -2.6 & 64921.0 & 5.38 & Plectin & NP_000436 & 17 & 29.0 & 67 \\
\hline 1446 & -2.6 & 17184.0 & 6.13 & Ubiquitin-conjugating enzyme E2N-like & NP_001013007 & 2 & 19.7 & 76 \\
\hline 894 & -3.0 & 80445.5 & 9.00 & Zinc finger protein ZNF45 & NP_003416 & 17 & 21.7 & 75 \\
\hline 759 & -3.3 & 43162.6 & 6.34 & Serpin peptidase inhibitor B7 & NP_001035237 & 10 & 20.5 & 48 \\
\hline 530 & -3.7 & 65783.3 & 8.11 & M6-kinase 3 & NP_060142 & 13 & 20.0 & 52 \\
\hline 1062 & -3.8 & 59753.1 & 7.50 & Cytokeratin type II & NP_004684 & 13 & 20.1 & 61 \\
\hline 1000 & -4.6 & 50348.3 & 9.45 & Regulator of $\mathrm{G}$ protein signalling 9 & NP_003826 & 13 & 25.8 & 64 \\
\hline 1376 & -12.1 & 17325.9 & 5.76 & Stathmin-1 & NP_981946 & 7 & 42.3 & 80 \\
\hline 430 & -13.2 & 60507.1 & 6.59 & Outer dense fiber of sperm tails 2-like isoform $b$ & _001007023 & 14 & 20.3 & 48 \\
\hline
\end{tabular}

isoform b (NP_001007023) were downregulated by a range from -2.3 to -13.2 . On the other hand, proteasome $26 \mathrm{~S}$ ATPase subunit 3 (NP_002795) and ubiquitin-conjugating enzyme E2N-like (NP_001013007) were upregulated by 4.7- and -2.6-fold, respectively. Thirdly, the regulatory proteins involved in cell signaling, differentiation and transcription were downregulated up to -12.1-fold. These proteins include stomatin (EPB72)-like 2 (NP_038470), M6-kinase 3 (NP_060142), regulator of G protein signaling 9 (NP_003826), stathmin-1 (NP_981946) and zinc finger protein ZNF45 (NP_003416) and Vav3 protein. However, the Vav3-specific antibody could not distinguish two forms from each other (Fig. 4A). Based on the location of spot, the protein was tentatively assigned as Vav3.1 ((NP_001073343). Vav3 is highly homologous to Vav1 and Vav2 both in mRNA sequence and amino acid sequence, whereas Vav3.1 is only composed of 287 amino acid residues, corresponding to the peptide sequence of two $\mathrm{SH} 3$ domains and one $\mathrm{SH} 2$ domain at the C-terminus of Vav3 $(41,42)$. Although no direct evidence shows that serpin peptidase inhibitor B7 (NP_001035237) and peroxiredoxin 6 (NP_004896) are directly involved in cell signal transduction, the cellular levels of these two proteins were also decreased respectively by 3.3- and 2.3fold in response to astragaloside IV. Collectively, our results suggest that astragaloside IV may affect cell proliferation and differentiation through decreasing the expression of some important regulatory proteins.

Verification of Vav3.1 expression. The protein spots corresponding to Vav3.1 were carefully compared by amplifying the protein image (Fig. 4B). To verify the suppression of Vav3.1 by astragaloside IV, the cellular proteins obtained from astragaloside IV-treated and untreated cells were resolved by $12 \%$ SDS-PAGE. The proteins in the gel were transferred 
A

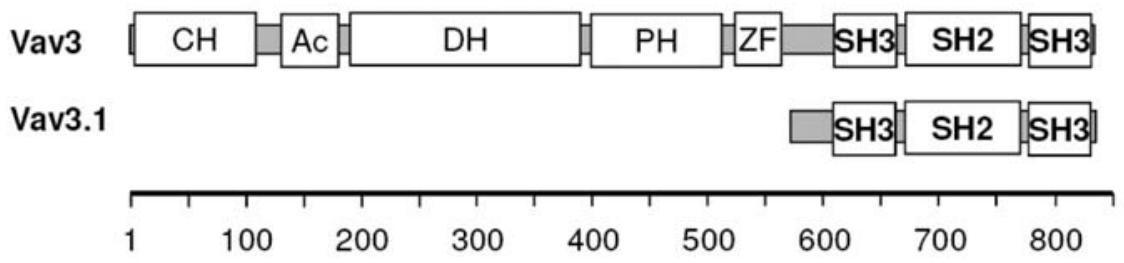

B

C
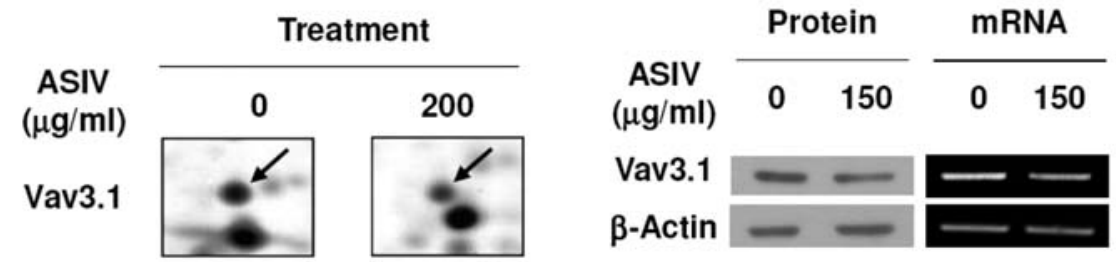

Figure 4. Verification of oncogenic Vav3.1 expression. (A) Illustration of the domain structures of Vav3 isoforms, Vav3 and Vav3.1, respectively. Abbreviations of the structure domains are referred to $\mathrm{CH}$, calponin-homology domain; Ac, acidic motif; DH, DBL-homology domain; PH, pleckstrinhomology domain; ZF, zinc-finger domain; PR, proline-rich region; SH3, SRC-homology 3 domain and SH2, SRC-homology 2 domain. The ruler indicates the length of peptide chain. (B) Magnified images of protein spots corresponding to Vav3.1. The oblique arrows indicate the protein spots corresponding to Vav3.1 in astragaloside IV-treated and control samples. (C) Detection of Vav3.1 expression by Western blotting and RT-PCR. HepG2 cells were treated with astragaloside IV $(150 \mu \mathrm{g} / \mathrm{ml})$ or vehicle only at $37^{\circ} \mathrm{C}$ for $24 \mathrm{~h}$. For Western blot analysis, the cell lysates were resolved by $12 \%$ SDS-PAGE and subsequently transferred onto PVDF membrane. The membrane was probed with rabbit anti-Vav3 or rabbit anti-B-actin antibodies. The bound antibodies were detected by goat anti-rabbit IgG-HRP conjugates and visualized by ECL reaction. For RT-PCR analysis, the total RNAs isolated from the cells were converted to complementary DNA templates. The primers specific for Vav3.1 and B-actin were used to amplify the DNA sequences encoding Vav3.1 and B-actin, respectively. PCR products were analyzed by $1.2 \%$ agarose gel electrophoresis.

onto PVDF membrane, the membrane was subsequently probed by specific antibodies against Vav3 and B-actin, respectively (Fig. 4C). Consistent with the results of previous 2D-electrophoresis analysis (Fig. 3), the antibody detected a peptide band, which matched the protein Vav3.1 (NP_001073343) in size (Fig. 4C). The signal intensity of Vav3.1 spot was decreased by 1.7-fold in astragaloside IVtreated sample compared with that of control sample. In order to overcome the difficulty of distinguishing Vav3 isoforms by using Vav3-specific polyclonal antibodies, oligonucleotide primers were specifically designed for the major transcript vav3.1 (NM_001079874) encoding Vav3.1 isoform. The PCR products are shown in Fig. 4C, in which Vav3.1 mRNA was clearly down-regulated by astragaloside IV. Such a result was highly consistent with the previous DNA microarray analysis (38). Thus, suppression of oncogenic Vav3.1 expression may represent a key mechanism underlying the action of astragaloside IV.

Regulation of Vav3.1 expression by astragaloside IV. Using a specific antibody, a panel of human cell lines were screened for Vav3.1 expression. As shown in Fig. 5, Vav3.1 was detected in HepG2, T47D, MB-AMD-231 PC-3 and HEK 293T cells at various levels, whereas no signal was detectable in MCF-7 and LNCaP cells, suggesting that Vav3.1 expression is cell-type dependent. Among three breast cancer cell lines, MB-AMD-132 and T47D are more invasive than MCF-7 (43). Similarly, PC-3 is a highly aggressive and androgenindependent prostate cancer cell line compared with LNCaP cells (44). Conceivably, Vav3.1 expression is positively

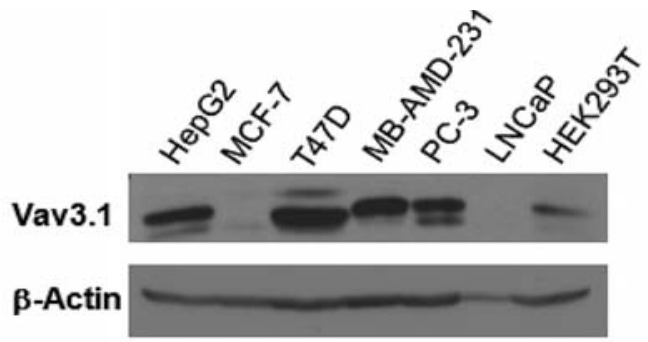

Figure 5. Detection of Vav3.1 expression in various cancer cell lines. The cells were cultured in the specific growth medium and harvested when the cell density reached $70-80 \%$ confluency. The cell lysates were resolved by $12 \%$ SDS-PAGE and subsequently transferred onto PVDF membrane. The membrane was probed with specific rabbit anti-Vav3 and anti- $\beta$-actin antibodies. The bound antibodies were detected by goat anti-rabbit IgGHRP conjugates and visualized by ECL kit.

correlated with the tumor aggressiveness. To understand the pharmacological regulation of Vav3.1 expression, HepG2 cells were used as the cellular detector and treated with astragaloside IV at the indicated doses of 0, 50, 100, 150 and $200 \mu \mathrm{g} / \mathrm{ml}$. As shown in Fig. 6A, Vav3.1 expression was suppressed by astragaloside IV in a dose-dependent manner. It is evident that Vav3.1 expression was strongly inhibited by astragaloside IV at the dose of $150 \mu \mathrm{g} / \mathrm{ml}$ and higher (Fig. 6B and C). We also observed a clear increase in the intracellular level of Vav3.1 with the prolonged cell culture under the normal conditions. These results stimulate us to postulate that suppression of Vav3.1 expression may be a key mechanism supporting the inhibition of tumorigenesis by astragaloside IV. 
A

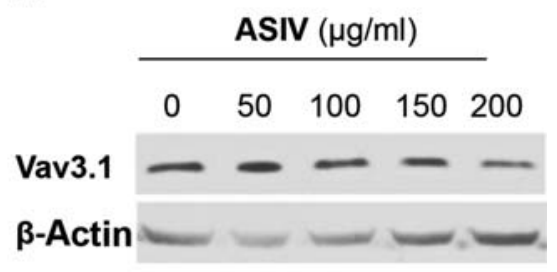

B
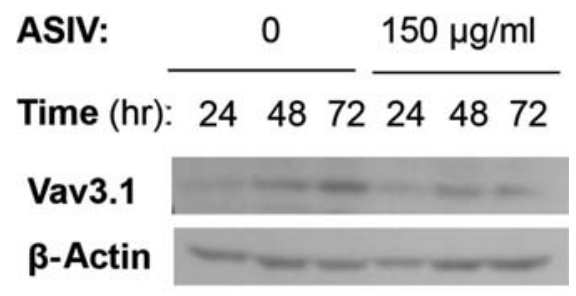

C

ASIV: 0 $150 \mu \mathrm{g} / \mathrm{ml}$

Time (hr): $24 \quad 48 \quad 72 \quad 24 \quad 48 \quad 72$

Vav3.1

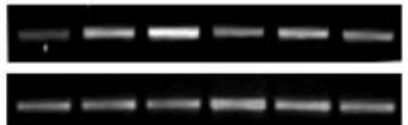

B-Actin $\longrightarrow$

Figure 6. Regulation of Vav3.1 expression by astragaloside IV in HepG2 cells. (A) Dose response. The cells were cultured in the specific growth medium and harvested when the cell density reached $70-80 \%$ confluency. The cell lysates were resolved by $12 \%$ SDS-PAGE and subsequently transferred onto PVDF membrane. The membrane was probed with specific rabbit antiVav3 and anti- 3 -actin antibodies. The bound antibodies were detected by goat anti-rabbit IgG-HRP conjugates and visualized by ECL kit. (B) Time course. The cells were cultured in the specific growth medium and harvested when the cell density reached $70-80 \%$ confluence. The cell lysates were resolved by $12 \%$ SDS-PAGE and sub-sequently transferred onto PVDF membrane. The membrane was probed with specific rabbit anti-Vav3 and anti- $\beta$-actin antibodies. The bound antibodies were detected by goat antirabbit IgG-HRP conjugates and visualized by ECL kit. (C) Suppression of Vav3.1 expression is astragaloside IV-dependent. The cells were cultured in the specific growth medium and harvested when the cell density reached 70$80 \%$ confluence. The cell lysates were resolved by $12 \%$ SDS-PAGE and sub-sequently transferred onto PVDF membrane. The membrane was probed with specific rabbit anti-Vav3 and anti- $\beta$-actin antibodies. The bound antibodies were detected by goat anti-rabbit IgG-HRP conjugates and visualized by ECL kit.

\section{Discussion}

A wide range of phytochemicals are effective to prevent and inhibit tumor initiation, promotion and progression (45). Radix Astragali is an important medicinal herb widely used in traditional Chinese medicine to treat various cancers (31). We recently observed that the major active compound astragaloside IV in the herb attenuated the ability of HepG2 cells to form cell foci and to grow in the anchorage-independent manner, suggesting a novel anticancer mechanism for this group of triterpenoids. The objective of this study was to further investigate the effects of astragaloside IV on protein expression in HepG2 cells. The proteins mostly affected by astragaloside IV were identified by MALDI/MS technology and their biological functions were annotated against public protein databases. According to our proteomic analysis, astragaloside IV affected the expression of a panel of proteins, especially the proteins involved in the stress response, protein biosynthesis and cell signaling pathways. Their associations with oncogenic transformation are discussed.

First of all, most of the significantly regulated proteins by astragaloside IV are involved in the cellular response to stress. Stress proteins include glucose-regulated protein BiP/GRP78 (NP_005338), heat shock $70 \mathrm{kDa}$ protein 2 (NP_068814), HSPA1A protein (NP_005336) and HSPA8 protein (NP_006588). Chaperon containing TCP1, subunit $8(\theta)$ (NP_006576) is not fully characterized, but likely to be a stress protein. When the cells are challenged by various stresses, heat shock proteins are coordinately upregulated whereas many other genes that were active before the metabolic insult are downregulated $(46,47)$. Heat shock proteins and other stress proteins normally function as molecular chaperones. These molecular chaperones interact with a panel proteins involved in several mechanisms, leading to the inhibition of the cellular inflammatory responses $(47,48)$. It is not known if astragaloside IV treatment imposes a kind of stress to the cells, the increased gene expression of stress proteins is critical to limit cell damage and facilitate the continued cell survival.

Secondly, our results also suggested that translation may be a key biological process targeted by astragaloside IV. Translation initiation enables the generation of functional gene products and thus plays a key role in the overall process of gene expression. In translation, messenger RNA (mRNA) is decoded to produce a specific polypeptide or protein according to the rules specified by the genetic code (49). Translation occurs in the cytoplasm where the ribosomes are located. Protein synthesis proceeds in four phases: activation, initiation, elongation and termination (49). Eukaryotic translation initiation factors (IF) and other proteins facilitate the binding of the small subunit of the ribosome to 5 ' end of mRNA, a key event to initiate the synthesis of a protein. Translation initiation factor eIF2 is essential for the initiation of protein synthesis because it protects formylmethionyl-tRNA from spontaneous hydrolysis as well as promotes the binding of Met-tRNA complex to the $30 \mathrm{~S}$ ribosomal subunits. In addition, the factor eIF2 takes part in the hydrolysis of GTP during the formation of the $70 \mathrm{~S}$ ribosomal complex. Translation initiation factor eIF3 is the largest mammalian translation initiation factor consisting of at least eight subunits with the mass ranging from 35 to $170 \mathrm{kDa}$ (50). Factor eIF3 forms a complex with the 40S ribosome in an early step of translation initiation and promotes the pairing of initiation Met-tRNA and mRNA. Eukaryotic translation initiation factor 3 subunit $2 B$ is a $36-\mathrm{kDa}$ protein containing WD40 repeats. eIF3 subunit $2 \beta$ was found to be identical to TRIP-1, a phosphorylation substrate of the TGF- $\beta$ type II receptor $(50,51)$. Translation initiation has been a major target for drug development (52). A variety of antibiotics such as anisomycin, cycloheximide, chloramphenicol, tetracycline, streptomycin, erythromycin, puromycin have been used to disable or inhibit translation in protein biosynthesis (52). Therefore, the biological importance of the regulation of translation initiation factors by astragaloside IV should be further addressed. 
Thirdly, our results showed that astragaloside IV altered the cellular level of several proteins involved in the cell signaling (Table I). M6-kinase 3 (NP_060142) is referred to as transient receptor potential cation channel (TRPC), subfamily M, member 7, and functions as both an ion channel and a kinase (53). As a channel, M6-kinase capacitates calcium entry into the cell. As a kinase, it catalyzes the phosphorylation of itself and other substrates. Importantly, its kinase activity is necessary for channel function. Intracellular ATP stimulates its kinase activity but PIP(2) hydrolysis causes the inactivation of the channel $(54,55)$. Several studies demonstrated a role for this group of proteins in cell survival and proliferation $(56,57)$. Regulator of G protein signaling 9 (RGS9) (NP_003826) is a member of the signaling protein RGS family that promote the GTPase activity of the G-protein coupled receptor proteins (58). Expression of mutated RGS9 was found in patients with bradyopsia, a disease with slow photoreceptor deactivation (59). Stathmin-1 (NP_981946) is a ubiquitously expressed cytosolic phosphoprotein $(60,61)$. Just like an intracellular relay, stathmin 1 integrates the cellular signals to regulate microtubule filament system by destabilizing microtubules $(62,63)$. Consequently, it prevents assembly and promotes disassembly of microtubules. Zinc finger protein ZNF45 (NP_003416) is a zinc ion binding transcription factor and regulates gene transcription in a DNA-dependent fashion $(64,65)$. Serpin peptidase inhibitor B7 (NP_001035237) was isolated as a new serpin with megakaryocyte maturation activity (66). This serpin was upregulated in IgA nephropathy (67). Moreover, transgenic rats overexpressing such serpin were found to develop novel serpinopathy in kidney and pancreas (68). Thus, astragaloside IV should be evaluated for the potential in improving IgA nephropathy and serpinopathy.

Among the three members of the vav oncogene family, vav3 shares 40 and $77 \%$ identity with vav and vav2, respectively, at the nucleotide level (42). Similar to Vav1 and Vav2, Vav3 is composed of several structural domains such as calponin-homology $(\mathrm{CH})$ domain, acidic (Ac) motif, DBLhomology $(\mathrm{DH})$ domain, pleckstrin-homology $(\mathrm{PH})$ domain, zinc-finger (ZF) domain, proline-rich (PR) region, two SRChomology 3 (SH3) domains and SRC-homology 2 (SH2) domain (Fig. 4A). Unlike other Vav members, Vav3 exists in two isoforms, a full length Vav3 and a short form Vav3.1, respectively $(41,42)$. Genetically, Vav3.1 may be derived from either a partly spliced transcript vav3.1 or totally different gene under the control of novel promoter. In contrast to Vav3, Vav3.1 is only composed of 287 amino acid residues corresponding to the C-terminus of Vav3, which contains two SH3 domains and one SH2 domain (Fig. 4A) (41). Vav3 is overexpressed in various cancers and highly correlated with the progression of human cancers (15). Knock-down of Vav3 causes decreased receptor transactivation in cancer cells (19). As far as the role of Vav3 in the cell malignant transformation is concerned, the C-terminal SH3 domains appear to be indispensable to its cell transforming activity, whereas the $\mathrm{N}$-terminal domains of Vav3 play a rather negative role $(69,70)$. Due to the lack of GEF domain, Vav3.1 protein does not catalyze the exchange of GDP/GTP. The biological function of Vav3.1 needs to be well-characterized.
Conceivably, Vav3.1 protein may substitute for Vav3 in functions mediated by this domain and compete with Vav3 in functions mediated by $\mathrm{N}$-terminal motifs. Nevertheless, our results suggest that pharmacological suppression of Vav3.1 may lead to the inhibition of tumorigenesis.

To explore the potential of Vav3.1 as a molecular target in cancer therapy, the regulation of Vav3.1 expression should be characterized at transcriptional and post-transcriptional levels. Growth factors TGF- $\beta$ and EGF were found to down-regulate the expression of Vav3.1 transcript in HaCaT keratinocytes, indicating a growth factor regulated mechanism (41). Here we showed for the first time that astragaloside IV down-regulated both Vav3.1 mRNA and protein in a doseand time-dependent manner. Thus, our results suggest a novel mechanism to support pharmacological intervention of cell malignant transformation by traditional herbal medicines.

In conclusion, we demonstrated that astragaloside IV altered the expression levels of several biologically important proteins in human HepG2 cells. Suppression of Vav3.1 expression appeared to be highly correlated with the inhibition of the cellular malignant transformation by astragaloside IV. Thus, when the role of Vav3.1 in the inhibitory effect of astragaloside IV on tumor formation is further clarified, it should be possible to develop a novel astragaloside IV-based anticancer therapy.

\section{Acknowledgements}

We acknowledge Miss Yim-Hing Cheung for her excellent technical assistance. We also thank Dr Priscilla Leung and Mr Lawrence Luk from Genome Research Centre, University of Hong Kong, for their technical assistance with 2D SDSPAGE analysis and protein identification by MALDI/TOF mass spectrometry. This work was supported by the Dean's Fund for Traditional Chinese Medicine Program, Li Ka Shing Faculty of Medicine, and the Seed Funding for Basic Research, The University of Hong Kong.

\section{References}

1. Mills JJ, Jirtle RL and Boyer IJ: Mechanism of liver tumor promotion. In: Liver Regeneration and Carcinogenesis. Jirtle RL (ed.) Academic Press, San Diego, pp199-226, 1995.

2. Hueber AO and Evan GI: Traps to catch unwary oncogenes. Trends Genet 14: 364-367, 1998.

3. Lynch DH: Oncogenes and cancer. Am J Reprod Immunol Microbiol 15: 24-28, 1987.

4. Breuhahn K, Longerich T and Schirmacher P: Dysregulation of growth factor signaling in human hepatocellular carcinoma. Oncogene 25: 3787-3800, 2006.

5. Vogelstein B and Kinzler KW: Cancer genes and the pathways they control. Nat Med 10: 789-799, 2004.

6. Satoh T, Nakafuku M and Kaziro Y: Function of Ras as a molecular switch in signal transduction. J Biol Chem 267: 24149-24152, 1992.

7. Ramjaun AR and Downward J: Ras and phosphoinositide 3kinase: partners in development and tumorigenesis. Cell Cycle 6: 2902-2905, 2007.

8. Gulbins E, Schlottmann K, Brenner B, Lang F and Coggeshall KM: Molecular analysis of Ras activation by tyrosine phosphorylated Vav. Biochem Biophys Res Commun 217: 876-885, 1995.

9. Friedman E: The role of ras GTPase activating protein in human tumorigenesis. Pathobiology 63: 348-350, 1995.

10. Malaney $\mathrm{S}$ and Daly RJ: The ras signaling pathway in mammary tumorigenesis and metastasis. J Mammary Gland Biol Neoplasia 6: 101-113, 2001 
11. Waldmann V and Rabes HM: What's new in ras genes? Physiological role of ras genes in signal transduction and significance of ras gene activation in tumorigenesis. Pathol Res Pract 192: 883-891, 1996.

12. Feig LA: The many roads that lead to Ras. Science 260: 767-768, 1993.

13. Katzav S, Packham G, Sutherland M, Aroca P, Santos E and Cleveland JL: Vav and Ras induce fibroblast transformation by overlapping signaling pathways which require c-Myc function. Oncogene 11: 1079-1088, 1995.

14. Sachdev P, Zeng L and Wang LH: Distinct role of phosphatidylinositol 3-kinase and Rho family GTPases in Vav3-induced cell transformation, cell motility, and morphological changes. J Biol Chem 277: 17638-17648, 2002.

15. Dong Z, Liu Y, Lu S, et al: Vav3 oncogene is overexpressed and regulates cell growth and androgen receptor activity in human prostate cancer. Mol Endocrinol 20: 2315-2325, 2006.

16. Liu Y, Mo JQ, Hu Q, et al: Targeted overexpression of vav3 oncogene in prostatic epithelium induces nonbacterial prostatitis and prostate cancer. Cancer Res 68: 6396-6406, 2008.

17. Lee K, Liu Y, Mo JQ, Zhang J, Dong Z and Lu S: Vav3 oncogene activates estrogen receptor and its overexpression may be involved in human breast cancer. BMC Cancer 8: 158, 2008.

18. Liu BP and Burridge K: Vav2 activates Rac1, Cdc42, and RhoA downstream from growth factor receptors but not betal integrins. Mol Cell Biol 20: 7160-7169, 2000.

19. Lyons LS and Burnstein KL: Vav3, a Rho GTPase guanine nucleotide exchange factor, increases during progression to androgen independence in prostate cancer cells and potentiates androgen receptor transcriptional activity. Mol Endocrinol 20: 1061-1072, 2006.

20. Abe K, Rossman KL, Liu B, et al: Vav2 is an activator of Cdc42, Rac1, and RhoA. J Biol Chem 275: 10141-10149, 2000.

21. Movilla N, Dosil M, Zheng Y and Bustelo XR: How Vav proteins discriminate the GTPases Rac1 and RhoA from Cdc42. Oncogene 20: 8057-8065, 2001.

22. Hornstein I, Alcover A and Katzav S: Vav proteins, masters of the world of cytoskeleton organization. Cell Signal 16: 1-11, 2004.

23. Katzav S, Martin-Zanca D and Barbacid M: vav, a novel human oncogene derived from a locus ubiquitously expressed in hematopoietic cells. EMBO J 8: 2283-2290, 1989.

24. Khosravi-Far R, Chrzanowska-Wodnicka M, Solski PA, Eva A, Burridge $\mathrm{K}$ and Der CJ: Dbl and Vav mediate transformation via mitogen-activated protein kinase pathways that are distinct from those activated by oncogenic Ras. Mol Cell Biol 14: 6848-6857, 1994.

25. Inabe K, Ishiai M, Scharenberg AM, Freshney N, Downward J and Kurosaki T: Vav3 modulates $\mathrm{B}$ cell receptor responses by regulating phosphoinositide 3-kinase activation. J Exp Med 195: 189-200, 2002

26. Hu P, Margolis B and Schlessinger J: Vav: a potential link between tyrosine kinases and ras-like GTPases in hematopoietic cell signaling. Bioessays 15: 179-183, 1993.

27. Zeng L, Sachdev P, Yan L, et al: Vav3 mediates receptor protein tyrosine kinase signaling, regulates GTPase activity, modulates cell morphology, and induces cell transformation. Mol Cell Biol 20: 9212-9224, 2000 .

28. Whitehead IP, Campbell S, Rossman KL and Der CJ: Dbl family proteins. Biochim Biophys Acta 1332: F1-F23, 1997.

29. Schmidt A and Hall A: Guanine nucleotide exchange factors for Rho GTPases: turning on the switch. Genes Dev 16: 1587-1609, 2002.

30. Etienne-Manneville S and Hall A: Rho GTPases in cell biology. Nature 420: 6629-635 2002.

31. Wang GL, Chen CB, Gao JM, Ni H, Wang TS and Chen L: [Investigation on the molecular mechanisms of anti-hepatocarcinoma herbs of traditional Chinese medicine by cell cycle microarray]. Zhongguo Zhong Yao Za Zhi 30: 50-54, 2005.

32. Krasteva IN, Toshkova RA and Nikolov SD: Protective effect of Astragalus corniculatus saponins against myeloid graffi tumour in hamsters. Phytother Res 18: 255-257, 2004.

33. Tin MM, Cho CH, Chan K, James AE and Ko JK: Astragalus saponins induce growth inhibition and apoptosis in human colon cancer cells and tumor xenograft. Carcinogenesis 28: 1347-1355, 2007.

34. Zhao KW and Kong HY: [Effect of Astragalan on secretion of tumor necrosis factors in human peripheral blood mononuclear cells]. Zhongguo Zhong Xi Yi Jie He Za Zhi 13: 263-265, 1993.
35. Wang DC: [Influence of Astragalus membranaceus (AM) polysaccharide $\mathrm{FB}$ on immunologic function of human periphery blood lymphocyte]. Zhonghua Zhong Liu Za Zhi 11: 180-183, 1989.

36. Kim HS, Lee EH, Ko SR, Choi KJ, Park JH and Im DS: Effects of ginsenosides $\mathrm{Rg} 3$ and $\mathrm{Rh} 2$ on the proliferation of prostate cancer cells. Arch Pharm Res 27: 429-435, 2004.

37. Bradford MM: A rapid and sensitive method for the quantitation of microgram quantities of protein utilizing the principle of protein-dye binding. Anal Biochem 72: 248-254, 1976.

38. Rong J, Tilton R, Shen J, et al: Genome-wide biological response fingerprinting (BioReF) of the Chinese botanical formulation ISF-1 enables the selection of multiple marker genes as a potential metric for quality control. J Ethnopharmacol 113: 35-44, 2007.

39. Wang LH: Molecular signaling regulating anchorage-independent growth of cancer cells. Mt Sinai J Med 71: 361-367, 2004.

40. Okada M, Jang SW and Ye K: Ebp1 association with nucleophosmin/B23 is essential for regulating cell proliferation and suppressing apoptosis. J Biol Chem 282: 36744-36754, 2007.

41. Trenkle T, McClelland M, Adlkofer K and Welsh J: Major transcript variants of VAV3, a new member of the VAV family of guanine nucleotide exchange factors. Gene 245: 139-149, 2000.

42. Movilla N and Bustelo XR: Biological and regulatory properties of Vav-3, a new member of the Vav family of oncoproteins. Mol Cell Biol 19: 7870-7885, 1999.

43. Lacroix M and Leclercq G: Relevance of breast cancer cell lines as models for breast tumours: an update. Breast Cancer Res Treat 83: 249-289, 2004

44. Sanchez AM, Sanchez MG, Malagarie-Cazenave S, Olea N and Diaz-Laviada I: Induction of apoptosis in prostate tumor PC-3 cells and inhibition of xenograft prostate tumor growth by the vanilloid capsaicin. Apoptosis 11: 89-99, 2006.

45. Surh YJ: Cancer chemoprevention with dietary phytochemicals. Nat Rev Cancer 3: 768-780, 2003.

46. Schlesinger MJ: Heat shock proteins. J Biol Chem 265 : 12111-12114, 1990 .

47. Arya R, Mallik M and Lakhotia SC: Heat shock genes - integrating cell survival and death. J Biosci 32: 595-610, 2007.

48. Pittet JF, Lee H, Pespeni M, O'Mahony A, Roux J and Welch WJ: Stress-induced inhibition of the NF-kappaB signaling pathway results from the insolubilization of the IkappaB kinase complex following its dissociation from heat shock protein 90. J Immunol 174: 384-394, 2005.

49. Stanley WM Jr, Salas M, Wahba AJ and Ochoa S: Translation of the genetic message: factors involved in the initiation of protein synthesis. Proc Natl Acad Sci USA 56: 290-295, 1966.

50. Asano K, Kinzy TG, Merrick WC and Hershey JW: Conservation and diversity of eukaryotic translation initiation factor eIF3. J Biol Chem 272: 1101-1109, 1997.

51. Chen RH, Miettinen PJ, Maruoka EM, Choy L and Derynck R: A WD-domain protein that is associated with and phosphorylated by the type II TGF-beta receptor. Nature 377: 548-552, 1995.

52. Galicia-Vazquez G, Lindqvist L, Wang X, Harvey I, Liu J and Pelletier J: High-throughput assays probing protein-RNA interactions of eukaryotic translation initiation factors. Anal Biochem 384: 180-188, 2009.

53. Runnels LW, Yue L and Clapham DE: TRP-PLIK, a bifunctional protein with kinase and ion channel activities. Science 291: 1043-1047, 2001.

54. Nadler MJ, Hermosura MC, Inabe K, et al: LTRPC7 is a Mg.ATP-regulated divalent cation channel required for cell viability. Nature 411: 590-595, 2001.

55. Runnels LW, Yue L and Clapham DE: The TRPM7 channel is inactivated by $\operatorname{PIP}(2)$ hydrolysis. Nat Cell Biol 4: 329-336, 2002.

56. Jiang J, Li MH, Inoue K, Chu XP, Seeds J and Xiong ZG: Transient receptor potential melastatin 7-like current in human head and neck carcinoma cells: role in cell proliferation. Cancer Res 67: 10929-10938, 2007.

57. Wykes RC, Lee M, Duffy SM, Yang W, Seward EP and Bradding P: Functional transient receptor potential melastatin 7 channels are critical for human mast cell survival. J Immunol 179: 4045-4052, 2007.

58. Zhang K, Howes KA, He W, et al: Structure, alternative splicing, and expression of the human RGS9 gene. Gene 240: 23-34, 1999.

59. Nishiguchi KM, Sandberg MA, Kooijman AC, et al: Defects in RGS9 or its anchor protein R9AP in patients with slow photoreceptor deactivation. Nature 427: 75-78, 2004. 
60. Ferrari AC, Seuanez HN, Hanash SM and Atweh GF: A gene that encodes for a leukemia-associated phosphoprotein (p18) maps to chromosome bands 1p35-36.1. Genes Chromosomes Cancer 2: 125-129, 1990.

61. Melhem RF, Zhu XX, Hailat N, Strahler JR and Hanash SM: Characterization of the gene for a proliferation-related phosphoprotein (oncoprotein 18) expressed in high amounts in acute leukemia. J Biol Chem 266: 17747-17753, 1991

62. Sobel A: Stathmin: a relay phosphoprotein for multiple signal transduction? Trends Biochem Sci 16: 301-305, 1991.

63. Olsen JV, Blagoev B, Gnad F, et al: Global, in vivo, and sitespecific phosphorylation dynamics in signaling networks. Cell 127: 635-648, 2006.

64. Thiesen HJ: Multiple genes encoding zinc finger domains are expressed in human T cells. New Biol 2: 363-374, 1990.

65. Shannon M and Stubbs L: Analysis of homologous XRCC1linked zinc-finger gene families in human and mouse: evidence for orthologous genes. Genomics 49: 112-121, 1998.
66. Tsujimoto M, Tsuruoka N, Ishida N, et al: Purification, cDNA cloning, and characterization of a new serpin with megakaryocyte maturation activity. J Biol Chem 272: 15373-15380, 1997.

67. Miyata T, Nangaku M, Suzuki D, et al: A mesangiumpredominant gene, megsin, is a new serpin upregulated in IgA nephropathy. J Clin Invest 102: 828-836, 1998.

68. Inagi R, Nangaku M, Usuda N, et al: Novel serpinopathy in rat kidney and pancreas induced by overexpression of megsin. J Am Soc Nephrol 16: 1339-1349, 2005.

69. Abe K, Whitehead IP, O'Bryan JP and Der CJ: Involvement of $\mathrm{NH}(2)$-terminal sequences in the negative regulation of Vav signaling and transforming activity. J Biol Chem 274: 30410-30418, 1999.

70. Groysman M, Nagano M, Shaanan B and Katzav S: Mutagenic analysis of Vav reveals that an intact SH3 domain is required for transformation. Oncogene 17: 1597-1606, 1998. 